University of Wollongong

Research Online

Faculty of Engineering - Papers (Archive)

Faculty of Engineering and Information

Sciences

$1-1-2012$

\title{
Spatial dispersion management in three-dimensional drawn magnetic metamaterials
}

\author{
Alessandro Tuniz \\ University of Sydney \\ Benjamin Pope \\ University of Sydney \\ Alexander Argyros \\ University of Sydney \\ Simon Fleming \\ University of Sydney \\ Anna Wang \\ University of Sydney
}

See next page for additional authors

Follow this and additional works at: https://ro.uow.edu.au/engpapers

Part of the Engineering Commons

https://ro.uow.edu.au/engpapers/5312

\section{Recommended Citation}

Tuniz, Alessandro; Pope, Benjamin; Argyros, Alexander; Fleming, Simon; Wang, Anna; Large, Maryanne C. J; Pogson, Elise M.; Lewis, Roger A.; Bendavid, Avi; and Kuhlmey, Boris T.: Spatial dispersion management in three-dimensional drawn magnetic metamaterials 2012, 1-2.

https://ro.uow.edu.au/engpapers/5312

Research Online is the open access institutional repository for the University of Wollongong. For further information contact the UOW Library: research-pubs@uow.edu.au 


\section{Authors}

Alessandro Tuniz, Benjamin Pope, Alexander Argyros, Simon Fleming, Anna Wang, Maryanne C. J Large, Elise M. Pogson, Roger A. Lewis, Avi Bendavid, and Boris T. Kuhlmey 


\title{
Spatial Dispersion Management in Three-Dimensional Drawn Magnetic Metamaterials
}

\author{
Alessandro Tuniz ${ }^{1}$, Benjamin Pope ${ }^{1}$, Alexander Argyros ${ }^{1}$, Simon Fleming ${ }^{1}$, Anna Wang ${ }^{1}$, \\ Maryanne C. J. Large ${ }^{1}$, Elise M. Pogson ${ }^{2}$, Roger A. Lewis ${ }^{2}$, Avi Bendavid ${ }^{3}$, and Boris T. Kuhlmey ${ }^{1}$ \\ ${ }^{1}$ Institute of Photonics and Optical Science (IPOS), School of Physics, University of Sydney, NSW, 2006, Australia \\ ${ }^{2}$ School of Engineering Physics, University of Wollongong, NSW, Australia \\ ${ }^{3}$ Commonwealth Scientific and Industrial Research Organization, Materials Science and Engineering, PO Box 218 Lindfield, Sydney, NSW, 2070 \\ Australia \\ Authore-mail address: alessandro.tuniz@sydney.edu.au
}

\begin{abstract}
We characterize resonances of 3D fiber metamaterials under transmittance at oblique incidence. The resonance frequency of longitudinally invariant resonators increases with the incident angle, while the resonance of disconnected resonators does not.

OCIS codes: (160.2290) Fiber materials; (160.3918) Metamaterials.
\end{abstract}

\section{Introduction}

Electromagnetic metamaterials attract much attention, providing optical properties that are not found in nature by arranging artificial resonant metal-dielectric structures on the sub-wavelength scale [1]. Recently, fiber drawing has emerged as a means of inexpensively fabricating bulk 3-dimensional metamaterials [2-4]. This process has so far allowed the production of continuous metal wires down to the micrometer [2] and nanometer [5] scale (allowing tailoring the effective permittivity), as well as longitudinally invariant metallic split-ring resonators on the microscale [3,4] (allowing tailoring the magnetic permeability). Previously [3], we fabricated U-shaped longitudinally invariant magnetic resonators in fiber form that possessed magnetic resonances near $0.35 \mathrm{THz}$ at normal incidence. Here we extend this procedure, using laser-ablation to break the longitudinal invariance, effectively producing 3-dimensional patterned arrays of on-fiber resonators. We measure the resonant transmittance properties of our fiber resonators as a function of incident angle using $\mathrm{THz}$ spectroscopy. We find that for the 3D (patterned) arrays the resonance does not shift as a function of angle, in contrast to the longitudinally invariant (unpatterned) samples. Our experimental results are in good agreement with three-dimensional finite element simulations.

\section{Fabrication and Characterization}

The fabrication procedure for our unpatterned samples is presented in Ref. [3]: a square preform of Zeonex [6] polymer is drawn to fiber with $100 \mu \mathrm{m}$ width, spooled onto a cylinder, and placed in a sputtering unit, where a $\sim 250 \mathrm{~nm}$ silver coating is applied on three sides to form U-shaped square resonators. This fiber is then re-spooled to produce a single flat metamaterial fiber array, supported in a frame. In this work, we used laser ablation to periodically remove $\sim 20 \mu \mathrm{m}$ portions of the metal coating on the three sides [Fig. 1 (a)], breaking the longitudinal invariance, and leaving three-dimensional resonators with a width of $10 \mu \mathrm{m}$, patterned on the fiber. A top-view optical microscope image of the resulting patterned and unpatterned portion of the samples is shown in Fig. 1(b). We first measured the transmittance under TE polarization (electric field directed along the fibers). All measurements were performed between $0.2-1 \mathrm{THz}$ using terahertz time domain spectroscopy. The results

a)

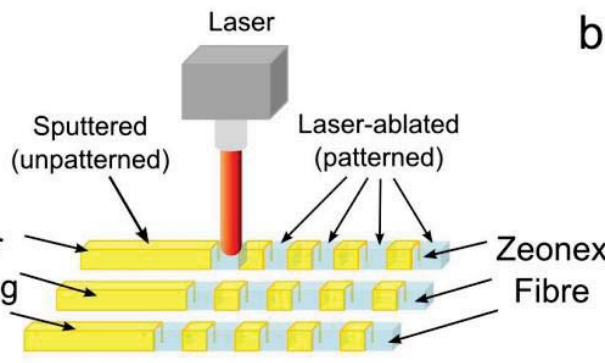

b)

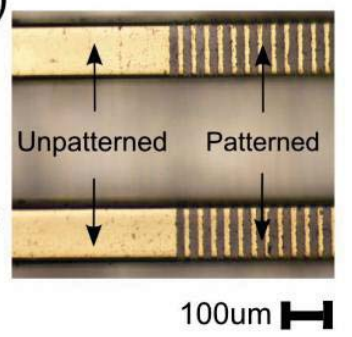

c)

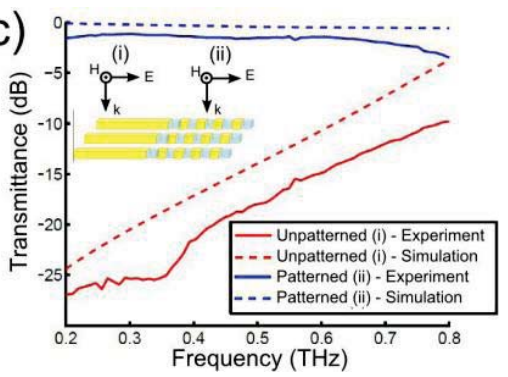

Fig.1: (a) Schematic of the laser-ablation procedure for producing 3-dimensional (patterned) resonators from longitudinally invariant (unpatterned) resonators. (b) Optical microscope image of the patterned and unpatterned fibers. (c) Measured and simulated transmittance for patterned and unpatterned samples for TE polarization (electric field directed along the fibers). 
are shown in Fig. 1(c) (solid lines.) Note that for the unpatterned portion of the sample, the array behaves like a high-pass filter due to the electric currents induced in the metal by the electric field, which is typical of subwavelength metallic gratings $[1,2]$. In contrast, the electric field is transmitted in the patterned portion of the sample, confirming that our fibers have been ablated on three sides, and that the resonators are electrically disconnected from each other. This is in good agreement with COMSOL finite element calculations (dashed lines).

a)

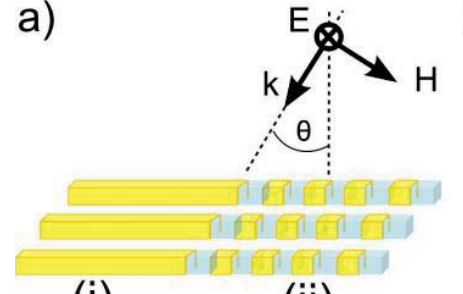

(i) b)i)

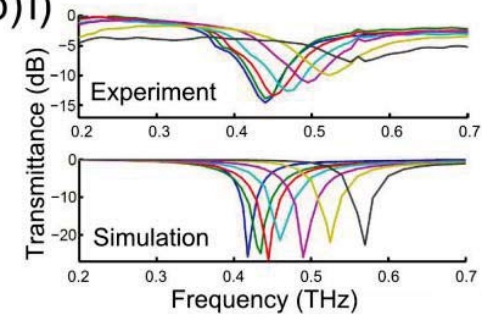

ii)

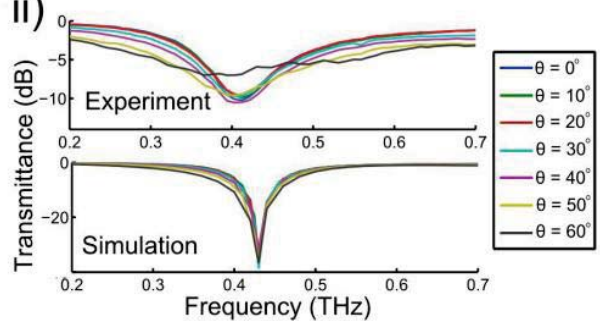

Fig.2: (a) Schematic of the transmittance experiment under TM polarization and oblique incidence. (b) Experimental and numerical spectral transmittance for different angles of incidence $\theta$ for (i) the unpatterned and (ii) the patterned portion of the sample.

We experimentally characterized the transmittance for the patterned and unpatterned samples under TM polarization (electric field perpendicular to the fibers) for different illumination angles $\theta$, as shown in Fig. 2(a). The results are shown in Fig. 2(b)(i) for the unpatterned samples and Fig. 2(b)(ii) for the patterned samples. Dips in transmission are associated with a strong magnetic resonance and thus strong variations in the effective magnetic response [3]. We observe that the resonant frequency for the unpatterned portion of the sample increases as the angle $\theta$ increases, thereby exhibiting strong spatial dispersion, which is a well-known issue in the case of wire metamaterials [7], but to our knowledge has not yet been studied in longitudinally invariant resonators. In contrast, the resonant frequency for the patterned fibers remains unchanged as the angle $\theta$ increases, in agreement with other oblique-incidence transmittance studies in split-ring resonators [8]. We model our fiber arrays using a 3dimensional finite element method (COMSOL), and obtain transmittance from scattering matrix parameters; the simulated geometry included a $100 \mu \mathrm{m}$ square Zeonex [6] fiber, coated by $1 \mu \mathrm{m}$ of silver on three sides, within a $300 \mu \mathrm{m}$-wide unit cell with Floquet boundary conditions to account for periodicity and changes in the incident angle. The simulation results, presented in Fig. 2(b), are in good agreement with experiment.

\section{Conclusion}

In conclusion, we have presented a procedure for fabricating 3-dimensional $\mathrm{THz}$ magnetic metamaterials in fiber form by laser ablating metal-coated square fibers. We characterized their resonances at oblique incidence, finding no strong dependence of resonant frequency on incident angle, in contrast to their longitudinally invariant counterparts, which exhibit strong spatial dispersion.

\section{Acknowledgements}

This work was performed in part at the Optofab node of the Australian National Fabrication Facility (ANFF) using Commonwealth and NSW State Government funding. We thank Dr Richard Lwin at the University of Sydney for assistance in drawing the fiber and Dr Benjamin Johnston at Macquarie University for assistance in laser-ablating the fiber. B.T.K. and A.A. acknowledge support from an Australian Research Council Future Fellowship and an Australian Research Fellowship, respectively.

[1] W. Cai and V. Shalaev, Optical Metamaterials: Fundamentals and Applications (Springer 2010 )

[2] A. Tuniz, B. T. Kuhlmey, R. Lwin, A. Wang, J. Anthony, R. Leonhardt, and S. C. Fleming, "Drawn metamaterials with plasmonic response at terahertz frequencies," Appl. Phys. Lett. 96, 191101 (2010)

[3] A. Wang, A. Tuniz, P. G. Hunt, E. M. Pogson, R. A. Lewis, A. Bendavid, S. C. Fleming, B. T. Kuhlmey, and M. C. J. Large, "Fiber metamaterials with negative magnetic permeability in the terahertz", Opt. Mat. Express 1, 115-120 (2011)

[4] A. Tuniz, A. Argyros, R. Lwin, S. C. Fleming, E. M. Pogson, E. Constable, R. A. Lewis, and B. T. Kuhlmey, "Stacked-and-drawn metamaterials with magnetic resonances in the terahertz range", Opt. Express 19, 17, 16480-16490 (2011).

[5] E. Badinter, A. Ioisher, E. Monaico, V. Postolache and I.M. Tiginyanu, "Exceptional integration of metal or semimetal nanowires in humanhair-like glass fiber", Mat. Lett. 64, 1902 (2010).

[6] J. Anthony, R. Leonhardt, A. Argyros, and M. C. J. Large, “Characterization of a microstructured Zeonex terahertz fiber,” J. Opt. Soc. Am. B 28, 1013-1018 (2011).

[7] P. A. Belov, R. Marqués, S. I. Maslovski, I. S. Nefedov, M. Silveirinha, C. R. Simovski, and S. A. Tretyakov, "Strong spatial dispersion in wire media in the very large wavelength limit", Phys. Rev. B 67, 113103 (2003)

[8] C. Menzel, R. Singh, C. Rockstuhl, W. Zhang, and F. Lederer, "Effective properties of terahertz double split-ring resonators at oblique incidence," J. Opt. Soc. Am. B, 26, B143-B147 (2009). 